\title{
Inflammation and autoimmunity in patients anti-neutrophil nuclear antibodies positive
}

\author{
Caterina Defendenti', Corrado Avarino', Mariella Saudelli', Enrico Magliano², \\ Raffaella Lopa ${ }^{3}$, Simone Saibeni ${ }^{4}$, Marco Pandolfi ${ }^{5}$ \\ 'Laboratorio analisi, Ospedale Fatebenefratelli, Milano \\ ${ }^{2}$ Dipartimento Sanità Pubblica, Microbiologia e Virologia, Università di Milano \\ ${ }^{3}$ Laboratorio di Citometria, Fondazione PO.MA.RE, Milano \\ ${ }^{4}$ Unità di Medicina Interna, Ospedale Fatebenefratelli, Milano \\ ${ }^{5}$ Unità di Pediatria, Ospedale Fatebenefratelli, Milano
}

Key Word: pANNA, Anti-neutrophil nuclear antibody, Epatite autoimmune, Colangite sclerosante

Infiammazione ed autoimmunità in due casi di colangite sclerosante

\section{SUMMARY}

Two patients, female, with overlap syndrome AlH/PSC and pANNA positive, was placed at two extremities of the bimodal curve that characterised the AlH I ${ }^{\circ}$ behaviour. Therefore they show difference of age (V.L. 76 years and E.G. 8 years) and different prognosis and therapy answer: the disease of the young patient has an aggressive outcome and fails the treatment more commonly. The more important theory to explain this different features was the "molecular footprint hypothesis" that considers decisive the type of HLA II".

The two patients considered have the same HLADR3. The serological analysis and the recent pathogenically orientations show that the local motives regulate the features of disease: in the child the inflammatory component prevails and maintains a chronic granulomatous aggression, whereas in the old patient the non specific autoimmune lgG prevails and drives a fibrosis evolution and immunosuppressive therapy response. The common positive pANNA can be expression of common chronic auto-inflammatory granulomatous origin.

\section{INTRODUZIONE}

L'epatite autoimmune (AIH) di tipo $\mathrm{I}^{\circ}$ viene diagnosticate in base allo scoring internazionale $\mathrm{e}$ presenta inequivocabili caratteristiche cliniche, biochimiche, istologiche e sierologiche $(4,5,18)$. Per definizione essa è una infiammazione epatocellulare cronica di causa sconosciuta caratterizzata istologicamente da epatite dell'interfaccia (piecemal necrosis) associata a ipergammaglobulinemia e autoanticorpi serici epato correlati.

Nella pratica clinica però non è raro osservare situazioni overlap, tra malattie ritenute a patogenesi differente. Un esempio non infrequente riguarda casi che manifestano aspetti di transizione tra epatite autoimmune $(\mathrm{AIH})$ di tipo $\mathrm{I}^{\circ}$ e colangite sclerosante (PSC).

La colangite sclerosante primitiva è probabilmente, secondo Chapman, una malattia immunomediata, piuttosto che immunologica. In effetti essa ha aspetti infiammatori granulomatosi cronici e potrebbe riconoscere come fattore scatenante l'ingresso di batteri o di altri metaboliti tossici nella circolazione portale in seguito ad alterazioni della parete intestinale. In effetti in letteratura la colangite sclerosante, tipica della giovane età, è spesso descritta come concomitante a malattie infiammatorie intestinali (13) facendo presupporre un momento patogenetico simile. Secondo alcuni autori questa associazione, che non sembra dovuta ad un comune rischio genetico (8), è tanto stretta da rasentare l' $80-100 \%(1,10,12,19)$. Una recente ipotesi $(10,12)$ ritiene che queste forme siano il risultato di un danneggiamento dell'immunità innata riguardante le cellule della barriera mucosa ed elementi cellulari, comprendenti neutrofili e macrofagi, che interagiscono in modo patologico con la flora enterica. Nel 100\% di dei casi di PSC sono presenti autoanticorpi definiti come pANNA (anti-neutrophil nuclear antibody), diretti verso i PMF e dei quali non è ancora stato identificato il pattern antigenico.

I quadri overlap AIH/PSC, sono spesso di difficile definizione e trattamento. Si discute ancora se se ci si trovi di fronte a due differenti malattie o a differenti manifestazioni della stessa malattia (11). I criteri diagnostici di questa sindrome over- 
lap sono: alte IgG, presenza di autoanticorpi, epatite dell'interfaccia e anormalità al colangiogramma. Anch'essi sono pANNA positivi.

I due casi descritti in questo lavoro, oltre a presentare gli aspetti tipici della sindrome overlap AIH/PSC, possono essere considerati rappresentativi dei due aspetti della curva bimodale con cui sembra esprimersi l'andamento dell'epatite autoimmune di tipo primo. Secondo studi $(2,3)$ condotti su popolazione anglosassone, l' $84 \%$ dei pazienti adulti caucasici con epatite autoimmune di tipi $\mathrm{I}^{\circ}$ provenienti dall'Inghilterra e dagli Stati Uniti, è HLA DR3, HLA DR4 o sia HLA DR3 che HLA DR4. Nell'ambito di questa popolazione vengono distinti due gruppi: (6) un gruppo di pazienti adulti ( $>60 \mathrm{aa}$ ) con alta frequenza di cirrosi alla presentazione rispetto ai pazienti con età inferiore a 30 anni ma con frequente remissione dopo trattamento steroideo e bassa incidenza di decessi causati dalla malattia epatica. Raramente necessitano di trapianto epatico. Essi presentano HLA DR4 più frequentemente che non i pazienti con età inferiore a 30 anni e, secondo gli autori, questa distinzione genetica può influenzare la frequenza di sviluppo di concomitante tiroidite o malattie reumatiche e la risposta al trattamento. Un secondo gruppo è costituito da pazienti più giovani, con fascia di età inferiore ai 30 anni, con HLADR3 e con remissione meno frequente, che spesso falliscono il trattamento e vanno sottoposti a trapianto di fegato.

Sulla base di queste osservazioni è stata formulata la "molecular footprint hypothesis" secondo la quale differenti alleli in differenti regioni geografiche e gruppi etnici possono favorire lo sviluppo della malattia attraverso la selezione naturale di pazienti con predisposizione genetica all'autoimmunità. La suscettibilità individuale prevista dagli alleli in una regione geografica può essere un "footprint".

Le due pazienti in esame hanno lo stesso fenotipo HLADR3. Interpretando i dati sierologici si è portati a ritenere, supportati dalle più recenti teorie patogenetiche, che il primum movens in ambedue i casi sia costituito da un difetto della reattività innata nei confronti di agenti infettivi che porta ad una flogosi non risolta. L'eccesso di antigene che si viene a creare stimola la formazione di autoanticorpi con varia specificità e bassa affinità. Nella forma dell'età adulta questa componente dell'immunità acquisita viene a prendere il sopravvento a livello locale.

\section{MATERIALI E METODI}

ANCA in immunofluorescenza indiretta:

Sono stati utilizzati vetrini di quattro pozzetti con adesi rispettivamente granulociti fissati in etano- lo, granulociti fissati in formalina, tessuto epatico e cellule HP2; diluizione 1/80 (Granulocytes Mosaic; Euroimmun).

\section{ELIA MPO, PR3}

Per la determinazioe delle due principali specificità anti neutrofili (MPO e PR3) è stato utilizzato un metodo immunoenzimatico con rilevazione in immunofluorescenza ELIA (Immunocap, Sweden).

\section{ANCA-Combi}

Test ELISA per la identificazione di ANCA atipici; riconosce elastasi, lattoferrina, lisozima, BPI, catepsina G (Chematil).

\section{Immunblot liver}

Identificazione in contemporanea di antigeni correlati con epatopatie adesi a striscia di cellulosa:

Gp210, sp100, LC1, M2, LKM1, PML SSA-Ro52 (Immunoblot Liver, Euroimmun);

\section{Citometria a flusso}

Il GIFT (Granulocyte Immunofluorescence Test) viene eseguito in citometria a flusso, utilizzando il FACSCalibur (Becton Dickinson) a quattro colori con software Cellquest.

Determinazione $H L A$ II ${ }^{\circ}$ con tipizzazione sierologia in linfocitotossicità.

\section{RISULTATI}

\section{A) Reattività autoanticorpale}

Paziente in età pediatrica: ANA speckled 1:160; ASMA G 1:160. Il tipo di pattern ed il basso titolo di questi autoanticorpi orientano verso una reattività aspecifica. Questa fluorescenza si è progressivamente ridotta fino a negativizzarsi nel corso della terapia corticosteroidea.

Paziente in età $>50$ aa: ANA nucleolare e nuclear lamins 1:320; ASMA G 1:320. I patterns evidenziati in questo caso non hanno subito modificazione nel corso della malattia e sono rimasti positivi anche dopo un anno di terapia immunosoppressiva (tabella1).

In ambedue i casi persiste tutt'ora una fluorescenza perinucleare a livello dei granulociti neutrofili fissati in etanolo, non supportata dalla positività ELISA per l'antigene tipico MPO. Alcuni autori (15) ritengono che la presenza di questi autoanticorpi nella sindrome overlap AIH/PSC sia del $100 \%$ e che in molti casi non sia possibile risalire al loro target antigenico; da qui la denominazione di anticorpi pANNA (anti-neutrophil nuclear antibody) come una entità a se stante.

La stretta associazione tra la flogosi cronica con 
forte presenza granulocitaria a carico dell'albero biliare e la positività pANNA potrebbe essere spiegata dai più recenti orientamenti sulla patogenesi delle malattie autoimmuni: durante le infezioni vengono prodotti numerosi autoanticorpi come risultato di attivazione non specifica (6) di cellule B autoreattive a bassa affinità. Si potrebbe ipotizzare che la presenza di antigeni in eccesso sia in grado di indurre uno stimolo policlonale (7, 14) con produzione di autoanticorpi a bassa affinità, diretti verso più componenti dei polimorfonucleati. Questo tipo di risposta poterebbe essere causa del rilievo di pANCA in IFI senza che necessariamente sia possibile identificare un target antigenico specifico.

La positività pANNA in fluorescenza permane anche dopo trattamento immunosoppressore come permane la flogosi cronica che la sostiene.

\section{B) Valutazione in ELISA degli ANCA atipici}

Il basso indice ottenuto in ELISA per ANCA atipici conosciuti non permette, in ambedue i casi, di sciogliere il dubbio sulla natura dell'antigene coinvolto e tuttora classificabile come pANNA. È probabile che questo marker comune sia espressione di una comune origine autoinfiammatoria (tabella 2).

\section{C) Confronto morfologico degli autoanticorpi anti citoplasma dei neutrofili}

Dal confronto del quadro IFI per ambedue i casi di overlap AIH/PSC non emergono differenze deci-

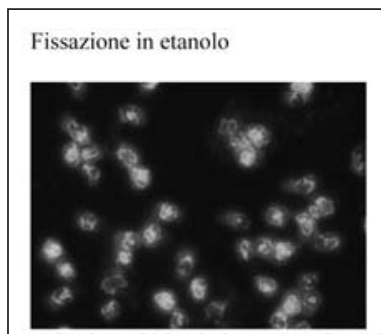

Fissazione in formalina

a) Paziente V.L. sindrome overlap AIH/PSC
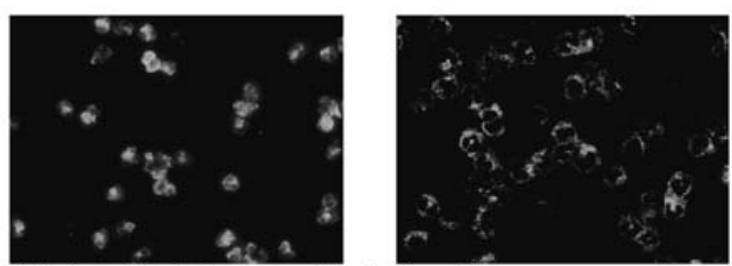

b) Paziente T.F. sindrome vasculitica MPO positiva

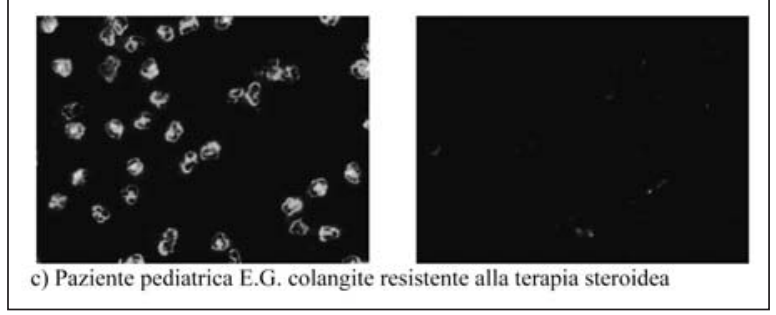

Figura I. Aspetto morfologico in IFI sive (figura I). Si possono evidenziare sfumature nelle caratteristiche della fluorescenza che risulta più o meno marcata intorno al nucleo. In formalina si ha negatività per la paziente E.G. e positività molto debole per la paziente V.L.

Le variazioni morfologiche possono dipendere dalla debole differenza nella composizione degli ANCA atipici che mostrano bassa positività per anti-BPI nella paziente pediatrica e bassa positività per autoanticorpi anti-elastasi (antigene strutturalmente simile a MPO, con positività citoplasmatica in formalina) e anti-catepsina $\mathrm{G}$ nella paziente adulta.

\section{D) Determinazione in citofluorimetria a flusso degli anticorpi anti-neutrofili}

Il test in citometria a flusso è routinariamente finalizzato alla ricerca $(9,17)$ di anticorpi anti neutrofili in pazienti con neutropenia di sospetta natura allo o autoimmune. Tale test evidenzia l'eventuale sia presenza di anticorpi diretti verso antigeni espressi sulla membrana cellulare dei neutrofili che la presenza di anticorpi nel siero del paziente.

In particolare si esegue il test diretto per la ricerca di anticorpi di classe IgG e IgM adesi alle cellule (neutrofili) del paziente; il test indiretto ricerca gli anticorpi circolanti di classe IgG.

Il test diretto prevede l'incubazione di circa 100$200 \times 10^{3}$ neutrofili del paziente con un antisiero Fab anti-human IgG e con anti-human IgM. In seguito si effettua una seconda incubazione con un anticorpo monoclonale per la marcatura specifica dei granulociti neutrofili (caratterizzati dell'espressione dell'antigene CD16). Mediante il citometro a flusso viene valutata la quantità di IgG o IgM adese a queste cellule.

Contestualmente si esegue il test indiretto che consiste nell'eseguire il cross-match del siero del paziente con 5-10 campioni di neutrofili di soggetti sani. Segue poi un'incubazione con l'antisiero anti-human IgG. I risultati dei test vengono quindi valutati in riferimento ai valori di controllo della giornata.

La paziente in età adulta presenta negatività per il test diretto ma una marcata positività nel test indiretto. Questo dimostra la presenza di anticorpi circolanti contro antigeni presenti sulla membrana dei polimorfonucleati (test indiretto positivo). Questi ultimi non sono però in grado di legarsi stabilmente sulla superficie delle cellule (test diretto negativo) e quindi di sostenere una granulocitopenia o manifestazioni sistemiche; essi probabilmente contribuiscono alla evoluzione fibrotica della flogosi epatica (tabella 3).

Nella paziente in età infantile invece non sono presenti autoanticorpi anti-neutrofili, né adesi alle cellule né circolanti. 
Tabella I. Schema riassuntivo degli aspetti autoimmunitari delle pazienti

\begin{tabular}{|l|l|l|l|l|}
\hline \multirow{2}{*}{} & \multicolumn{2}{|c|}{$\begin{array}{l}\text { PAZIENTE } \\
\text { PEDIATRICA }\end{array}$} & \multicolumn{2}{c|}{ PAZIENTE >50 ANNI } \\
\hline autoanticorpi & ALL'ESORDIO & $\begin{array}{l}\text { DOPO } \\
\text { TRATTAMENTO }\end{array}$ & ALL'ESORDIO & $\begin{array}{l}\text { DOPO } \\
\text { TRATTAMENTO }\end{array}$ \\
\hline ANA & $\begin{array}{l}1: 160 \\
\text { speckled }\end{array}$ & negativo & $\begin{array}{l}1: 320 \text { nuclear } \\
\text { lamins e nucleolare }\end{array}$ & $\begin{array}{l}1: 320 \text { nuclear lamins } \\
\text { e nucleolare }\end{array}$ \\
\hline ASMA & $1: 160$ & negativo & $1: 320$ & $1: 320$ \\
\hline APGA & negativo & negativo & negativo & negativo \\
\hline $\begin{array}{l}\text { AMA } \\
\text { negativo }\end{array}$ & negativo & negativo & negativo \\
\hline $\begin{array}{l}\text { LKM } \\
\text { negativo }\end{array}$ & negativo & negativo & negativo \\
\hline $\begin{array}{l}\text { LCI } \\
\text { negativo } \\
\text { BLOT }\end{array}$ & negativo & negativo & negativo & negativo \\
\hline $\begin{array}{l}\text { ANTI-MPO } \\
\text { Metodo ELIA }\end{array}$ & negativo & negativo & negativo & negativo \\
\hline $\begin{array}{l}\text { ANTI-PR3 } \\
\text { Metodo ELIA }\end{array}$ & negativo & negativo & negativo & negativo \\
\hline $\begin{array}{l}\text { ANCA } \\
+++\end{array}$ & pANCA & negativo & pANCA \\
+++ & +++ & \\
\hline
\end{tabular}

Tabella 2. Schema riassuntivo degli ANCA atipici valutati in ELISA

\begin{tabular}{|l|l|l|l|}
\hline & & Campione V.L. & Campione E.G. \\
\hline A & Miscela antigeni & Negativo & CUT-OFF \\
\hline B & PR3 & 0,9 & 0,5 \\
\hline C & MPO & 0,6 & 0,4 \\
\hline D & BPI & 0,6 & $\mathbf{1 , 4}$ \\
\hline E & Elastasi & $\mathbf{1 , 4}$ & 0,9 \\
\hline F & Catepsina G & $\mathbf{1 , 1}$ & 0,6 \\
\hline G & Lisozima & 0,8 & 0,7 \\
\hline H & Lattoferrina & 0,5 & 0,5 \\
\hline
\end{tabular}

Tabella 3. Schema riassuntivo della reattività antineutrofili in citometria a flusso

\begin{tabular}{|l|l|l|}
\hline $\begin{array}{l}\text { IgG-IgM antineutrofili } \\
\text { in citometria a flusso }\end{array}$ & PAZIENTE PEDIATRICA & PAZIENTE $>50$ aa \\
\hline TEST DIRETTO & NEGATIVO & NEGATIVO \\
\hline TEST INDIRETTO & NEGATIVO & $\begin{array}{l}\text { POSITIVO CONTRO TUTTE LE } \\
\text { SOSPENSIONI CELLULARI }\end{array}$ \\
\hline
\end{tabular}

\section{CONCLUSIONI}

Le due pazienti prese in esame sono ambedue HLA DR3 ma dal punto di vista clinico e prognostico appartengono ciascuna a uno dei differenti gruppi della curva bimodale. Ambedue presentano un impegno colangitico e sono positivi per anticorpi pANNA.

Analizzando le caratteristiche di questi due casi sembra emergere l'ipotesi della presenza di due fasi patogenetiche: una, quella in età infantile, in cui l'aspetto prevalente è di tipo infiammatorio e l'altra, in età adulta, in cui assume maggiore importanza la componente autoimmunitaria che mantiene però le caratteristiche di un aggressione locale senza assumere connotati sistemici.

In effetti nella forma pediatrica il trattamento immunosoppressore, solo corticosteroideo, non si è dimostrato clinicamente efficace (persistenza di transaminasi e gamma GT elevate): la negativizzazione dei parametri immunitari valutati in IFI ed ELISA (xANCA in IFI è l'unico parametro che persiste positivo) dopo terapia è stata accompagnata da un miglioramento clinico solo parziale.

La paziente in età adulta manifesta invece un overlap autoimmune: sono presenti autoanticorpi con patterns differenti (nuclear lamins, nucleolare, ASMA, anti antigeni di superfice dei neutrofili, anti elastasi, anti catepsina $\mathrm{G}$ ) la maggior parte dei quali rimane rilevabile in laboratorio anche dopo remissione sintomatologica in seguito a trattamento con corticosteroidi e azatioprina. L'esito è una evoluzione fibrotica.

Allo stato attuale delle nostre conoscenza la forma a prevalente componente infiammatoria è più difficilmente diagnosticabile e curabile.

La comune origine autoinfiammatoria potrebbe essere testimoniata dalla positività pANNA. È probabile che questo marker comune dia luogo ad espressioni patologiche diverse in relazione all'età delle pazienti con prevalente espressione infiammatoria nelle forma giovanile e progressivo maggiore coinvolgimento autoimmune nelle forme dell'adulto.

Il basso indice ottenuto in ELISA per ANCA atipici conosciuti e le caratteristiche in IFI non permettono, in ambedue i casi, di sciogliere il dubbio di un overlap tra le specificità riscontrate e un pattern sconosciuto e tuttora classificabile come pANNA (16). Nella forma in età adulta è però evidenziabile in citometria un parametro aggiuntivo costituito dalla presenza di anticorpi diretti contro marker di superficie dei granulociti neutrofili dotati si scarsa affinità; esso può essere interpretato come una conferma della tendenza alla estensione della risposta autoimmune verso determinanti antigenici sempre più vari senza che venga raggiunto un punto di rottura in grado di indurre una risposta sistemica.

I più recenti orientamenti sulla patogenesi delle malattie autoimmuni considerano fondamentale un'alterata capacità di risposta alle infezioni che induce un'importante flogosi cronica. Con il perdurare dello stimolo dell'immunità innata, vengo- 
no prodotti numerosi autoanticorpi come risultato di attivazione non specifica (6) di cellule B autoreattive a bassa affinità.

Si ipotizza che la presenza di antigeni in eccesso sia in grado di indurre uno stimolo policlonale (7, 14) con produzione di autoanticorpi a bassa affinità, diretti verso più componenti dei polimorfonucleati. Dal punto di vista laboratoristico sarebbe quindi possibile rilevare positività intensa pANCA senza poter identificare un singolo antigene coinvolto.

\section{BIBLIOGRAFIA}

1. Broomé U, Bergquist A. Primary sclerosing cholangitis, inflammatory bowel disease, and colon cancer Semin liver dis. 2006 feb; 26(1): 31-41.

2. Czaja AJ, Doherty DG, Donaldson PT. Genetic Bases of Autoimmune Hepatitis Digestive Diseases and Sciences. October 2002; 47(10): 2139-50.

3. Czaja AJ, Donaldson PT. Genetic susceptibilities for immune expression and live cell injury in autoimmune hepatitis Immunological. Reviews 2000; 174: 2509.

4. Czaja AJ. Diverse manifestations and evolving treatments of autoimmune hepatitis. Minerva Gastroenterol Dietol 2005; 51: 313-3.

5. Czaja AJ. Frequency and nature of the variant syndromes of autoimmune liver disease. Hepatology 1998; 28: 360-5.

6. Gross WL, Trabandt A, et al. Pathogenesis of Wegener's granulomatosis. Ann Med Interne (Paris). 1998 Sep; 149(5): 280-6.

7. Harper H, Cockwell $\mathrm{P}$, et al. Neutrophil priming and apoptosis in anti-neutrophil cytoplasmic autoantibody-associated vasculitis. Kidney International 2001;
59: 1729-38.

8. Karlsen TH, Hampe J, Wiencke K, et al. Genetic polymorfisms associated with inflammatory bowel disease do not confer risk from primary sclerosing cholangitis. Am J Gastroenterolog 2007; 102: 115-21.

9. Kiefhaefer K, et al. Detection of granulocyte antibodies by flow cytometry without the use of pure granulocyte isolates. Trasfusion 2000-vol. 40, supplement, $104 \mathrm{~S}$

10. Korzenik JR. Is Crohn's disease due to defective immunity? Gut 2007; 56: 2-5.

11. La patologia epatica autoimmune oggi: dalla diagnosi al trapianto. Riunione monotematica A.I.S.F. 2006. Bologna, 5-6 ottobre 2006.

12. Levine AD, Fiocchi C. Immunology of inflammatory bowel disease. Curr Opin Gastrienterol. 200 Jul; 16(4): 306-9.

13. Premoli A, Morello E, Bo S, Durazzo M. Diagnostic and Therapeutic questions in overlap syndromes of autoimmune hepatitis. Minerva Gastroenterol Dietol. 2007 Mar; 53(1): 79-82.

14. Soulas P, Woods A, et al. Autoantigen, innate immunità and $\mathrm{T}$ cells cooperate to break $\mathrm{B}$ cell tolerance during bacterial infection. J Clin Invest 2005; 115: 2257-67.

15. Terijung B. Eur J Med Res 2004; 9: 439-48.

16. Terjung B, Worman HJ, et al. Differentiation of antineutrophil nuclear antibodies in inflammatory bowel and autoimmune liver diseases from antineutrophil cytoplasmic antibodies (pANCA) using immunofluorescence microscopy; Clinical \& Experimental Immunology October 2001; 126(1): 37-46.

17. Trasfusion Medicine Methods for detection of neutrophil antigen/antibody reactions; Part VIII, 1976-1979.

18. Vergani D, Mieli Vergani G. Autoimmune hepatitis. Autoimmunity Reviews 2 (2003); 241-7.

19. Xiaofa Quin. Primary Sclerosing Cholangitis and Inflammatory Bowel Disease: Where is the link? American Journal of Gastroenterology 2007-1332. 